\title{
Epigenomic evolution in chronic lymphocytic leukaemia cells from patients treated with ibrutinib and showing disease progression
}

\section{Katie Holmes}

Leeds Institute of Cancer and Pathology

Ildar Sadreev

Leeds Institute of Cancer and Pathology

Charlotte Evans

Leeds Institute of Cancer and Pathology

Fraser Thomas

Leeds Institute of Cancer and Pathology

Andy Rawstron

Leeds Institute of Cancer and Pathology

Pete Hillmen

Leeds Institute of Cancer and Pathology

Darren James Newton ( $\boldsymbol{\nabla}$ d.j.newton@leeds.ac.uk)

University of Leeds https://orcid.org/0000-0002-0214-1486

Pascal Lefevre

Leeds Institute of Cancer and Pathology

Research

Keywords: CLL, ibrutinib, relapse, epigenome, plasticity

Posted Date: June 18th, 2020

DOl: https://doi.org/10.21203/rs.3.rs-33939/v1

License: (1) This work is licensed under a Creative Commons Attribution 4.0 International License.

Read Full License 


\section{Epigenomic evolution in chronic lymphocytic leukaemia}

\section{2 cells from patients treated with ibrutinib and showing}

\section{3 disease progression}

4 Katie B. Holmes ${ }^{1}$, Ildar I. Sadreev ${ }^{1}$, Charlotte L. Evans ${ }^{1}$, Fraser Thomas ${ }^{1}$, Andy C. Rawstron ${ }^{2}$,

$5 \quad$ Peter Hillmen ${ }^{1}$, Darren J. Newton ${ }^{1}$ and Pascal F. Lefevre ${ }^{1}$

6

$7{ }^{1}$ Division of Haematology and Immunology, Leeds Institute of Medical Research at St.

8 James's, University of Leeds, Leeds, UK; ${ }^{2}$ Haematological Malignancy Diagnostic Service

9 (HMDS), St. James's Institute of Oncology, Bexley Wing, Beckett Street, Leeds LS9 7TF, UK

11 Correspondent author: Dr Darren J. Newton, Division of Haematology and Immunology,

12 Leeds Institute of Medical Research, St. James's University Hospital, Leeds LS9 7TF UK,

13 Tel.: +44-113-3437645, Fax: +44-113-3438502, email: D.J.Newton@leeds.ac.uk

15 Running title: Epigenomic changes in ibrutinib-treated CLL cells 


\section{Abstract}

\section{Background}

The Bruton's tyrosine kinase (BTK) inhibitor ibrutinib provided a breakthrough in the treatment of chronic lymphocytic leukaemia (CLL), but cases of resistance are now emerging. Whilst resistance is commonly associated with mutations in BTK itself and the downstream signalling molecule PLC $\gamma 2$, this is not always the case. In a recent study, we have detected some epigenomic plasticity correlated with the dynamics of CLL cell response to ibrutinib. To understand the mechanisms mediating resistance in CLL, it is important to be able to determine whether the observed phenotypic changes are driven solely by resistance cues (e.g. clonal evolution, activation of signalling bypassing BTK inhibition), or if they could also be the consequence of unrelated events.

Methods

To answer to this question, we have monitored chromatin changes happening in response to ibrutinib from the start of treatment until relapse in CLL cells from both a patient carrying a previously identified BTK C481S mutation and from a relapsing patient, for whom none of the classical genetic lesions associated with ibrutinib relapse was detected.

$40 \quad$ Results

We established that the epigenome and gene expression in CLL cells from patients on

42 ibrutinib changes with time independently of disease progression and identified two patterns

43 of chromatin alterations, which are independent of resistance mechanisms; one ibrutinib-

44 dependent and the other related to quiescence. Furthermore, by defining the main characteristics of resistance-independent epigenomic plasticity and excluding them from the analysis, we identified candidate genes potentially mediating disease progression.

\section{Conclusion}


This two-step strategy could fundamentally alter the understanding of resistance to

50 treatment in CLL.

51

52 Key words: CLL, ibrutinib, relapse, epigenome, plasticity 


\section{Background}

Chronic lymphocytic leukaemia (CLL), the most frequent leukaemia in western countries, originates from clonal proliferating B-cells $(1,2)$. Treatment with the Bruton's Tyrosine Kinase (BTK) inhibitor ibrutinib has shown extremely promising results in front-line treatment as well as in relapsed/refractory $\operatorname{CLL}(3,4)$ and is now being tested in combination with other therapies (5-7). However, a significant number of cases of both primary and secondary resistance have been reported $(8,9)$ and when treatment is discontinued, longterm outcome is generally poor (10). Intensive efforts are undertaken to better understand the mechanism of response to ibrutinib and the development of drug resistance. In CLL cells, the initial response is a sharp downregulation of the NF-KB and BCR signalling pathways (11-14) and a reduced activity of lineage specific transcription factors (14). This is followed by ibrutinib-dependent lymphocytosis (15), cells from lymph nodes being expelled into the peripheral blood (PB). These cells are typically over-expressing genes related to proliferation pathways and cell cycle (16). Accordingly, epigenomic analysis of these cells reveals transient increases in histone $\mathrm{H} 3$ lysine 4 trimethylation (H3K4me3), a histone post-translational modification (PTM) associated with open chromatin at cis-elements targeted by lineage defining transcription factors providing a signature of activation (17). Longer exposure to ibrutinib induces erosion of CLL identity correlated with a quiescent-like gene expression signature (14), global loss of both H3K27 acetylation (activating) and trimethylation (repressive) marks (17). Furthermore, while H3K4me3 stays globally relatively stable with time on ibrutinib, this histone PTM is distinctly reduced in regions identified as bivalent $(\mathrm{H} 3 \mathrm{~K} 4 \mathrm{me} 3+/ \mathrm{H} 3 \mathrm{~K} 27 \mathrm{me} 3+)$ before treatment (17). All of these observations indicate an important epigenomic plasticity of CLL cells in response to ibrutinib treatment.

Relapsed CLL, after ibrutinib therapy, is commonly associated with genomic alterations in $B T K$ and its downstream target $P L C \gamma 2$. However, cases of progressive disease in which targeted sequencing is unable to explain resistance have also been reported (12) 
and activation of alternative signalling pathways that bypass BCR signalling might also play a

81 role in such process.

In the present analysis, we monitored chromatin structure evolution in CLL cells from patients treated with ibrutinib before and after disease progression (Supplementary tables 1 and 2). In contrast with genetic abnormalities, the specificity of the chromatin landscape for a given individual correlates with cell phenotype and is, consequently, relevant for this particular individual when compared to clinical outcome. This study, combined with gene expression analysis, suggests coordinated phenotypic variations of CLL cells independent of disease evolution, characteristic of either cell activation or quiescence, consistent with our previous observations (17), and not explained by technical variability. We also determined that WNT5A might participate in the mechanism of relapse in a patient with no detectable mutation of either the BTK or PLC $\gamma 2$ genes.

\section{Results}

\section{Chromatin structure varies with time in CLL cells from patients on ibrutinib}

We have previously analysed the evolution of the chromatin structure in CLL cells from patients enrolled in the IcICLLe trial, a feasibility study investigating the mechanism of action of ibrutinib. Patients received continuous oral therapy with ibrutinib (420 mg OD) and CLL cells were collected from PB before and at regular time points during treatment (17). From this trial, we identified a CLL patient (IbruR3) who progressed after 24 months of ibrutinib therapy (Supplementary Fig. S1A). We screened for previously identified mutations associated with relapse $(18,19)$. Interestingly, we detected the previously characterised BTK C481S mutation after 32 months, but not at month 30 even though the percentage of CLL cells in the PB was already notably increasing (Fig. 1A, Supplementary Fig. S1A). In addition, the level of $\mathrm{H} 3 \mathrm{~K} 27 \mathrm{me} 3$ and H3K27ac was low at 6 months post treatment initiation for this patient, in agreement with our previous observation showing global disappearance of both histone marks 
107 after months on ibrutinib (17) and was elevated at 33 months corresponding with progression.

108 The 9 months' time point showed a normal level of H3K27me3 as well as a slight and global

109 increase in H3K27ac compared with 3 months earlier (Fig. 1B and C, Supplementary Fig.

110 S1B). However, CLL cell count was decreasing at 9 months suggesting that the patient was

111 responding well to the treatment at that time (Supplementary Fig. S1A). Furthermore, we

112 compared this observation with the analysis of the chromatin structure of CLL cells obtained

113 from peripheral blood samples from a patient with acquired resistance to ibrutinib (IbruR1),

114 who was not enrolled in the trial. For this patient, we analysed chromatin changes in two

115 samples collected within an interval of 3 months, both after the diagnosis of relapse. PCR

116 screening for mutations including BTK C481S and the PLC $\gamma 2$ L845F, R665W and S707Y

117 mutations $(18,19)$ was negative for both time points. A genome-wide increase in the monitored

118 histone marks was observed in this patient for the first time point, however, a noticeable

119 reduction in both $\mathrm{H} 3 \mathrm{~K} 27 \mathrm{me} 3$ and $\mathrm{H} 3 \mathrm{~K} 27 \mathrm{ac}$ was observed 3 months later, suggesting that

120 disease progression might not be a continuous process (Fig. 1B and C, Supplementary Fig.

121 S1B and C). Altogether, these data suggested that some chromatin changes are dependent

122 and some independent of disease evolution.

\section{H3K27ac can vary independently of disease progression}

To further characterise the observed changes and determine if technical variability could be ruled out, we performed a quantitative analysis of H3K27ac evolution compared to before treatment. Loss or reduction in $\mathrm{H} 3 \mathrm{~K} 27 \mathrm{ac}$ was global and partially restored at 33 months compared to before treatment (Fig. 2A). Interestingly, the global change of H3K27ac peak intensity was comparable between IbruR3 before treatment and in the progressing sample

$130(33 \mathrm{M})$ and between the 3 months interval samples for IbruR1, suggesting a similar reduced 131 transcriptional activity in both later time points (Fig. 2A). 
135 the selected peaks, did not reveal any specific functional association for the $6 \mathrm{M}$ high peaks

136 but the 9M high fraction was enriched for genes of the canonical WNT and TGF $\beta$-signalling

137 pathways as well as genes associated with regulation of cell growth (Supplementary Fig. S2A

138 Supplementary Table 4). Next, we analysed the intersection between our peak libraries and

139 merged transcription factor ChIP-seq data obtained from a publicly available database

140 (ReMap). This analysis, referred to as REMI, calculates the number of cis-regulatory elements

141 enriched for a specific histone mark and containing a validated consensus sequence

142 corresponding to a specific transcription factor (17). This allowed us to generate signatures

143 associated with determined peak populations and to assess the relative variation of these

144 signatures when comparing one peak population to another (17). Using REMI analysis, we

145 identified an enrichment for peaks containing validated binding-sites for the mediator complex

146 (CDK8, MED12) and the Polycomb Receptor Complex 2 (PRC2) (EZH2, SUZ12) in the 9M

147 high peak population and for SOX2 and PRDM1 in the 6M high one (Fig. 2C).

148 The same analysis performed to compare the H3K27ac peak population between

149 IbruR1 and IbruR3 BT suggested that histone acetylation was increased for genes of the

150 stress activated signalling cascade or cell-cell adhesion in the nearest genes associated with

151 peaks high in IbruR1 or IbruR3 cells respectively (Supplementary Fig. S2B, Supplementary

152 table 4). Interestingly, EZH2 and SUZ12 functional binding sites were found in the decreased

153 H3K27ac peaks fraction and SOX2 in peaks enhanced in IbruR1 compared to IbruR3,

154 respectively (Fig. 2D). Moreover KLF4, which was among the genes with higher level of

$155 \mathrm{H} 3 \mathrm{~K} 27 \mathrm{ac}$ at associated cis-regulatory elements in IbruR3 compared to IbruR1, was also found

156 enriched in the IbruR3 high peak population (Fig. 2D, Supplementary table 4). Altogether

157 these data suggested that loss of H3K27ac at EZH2/SUZ12-associated peaks was correlated

158 with a gain in SOX2-associated peaks in these cells, such coordinated changes ruling out

159 technical artefacts.

160

161 Changes in H3K4me3 peak number follow H3K27ac evolution 
We have shown previously that $\mathrm{H} 3 \mathrm{~K} 4 \mathrm{me} 3$ was progressively disappearing at bivalent

163 cis-elements and transiently increasing at lineage specific cis-elements (LSCE) (17). The latter

164 changes were correlated with cells entering the peripheral blood from the proliferation centre,

165 which could be labelled as activated-CLL cells (17).

166 Here, comparing H3K4me3 peak number evolution between our different time points,

167 we observed dynamic variations concordant with those seen for H3K27ac. These variations reached a maximum of $16 \%$ peak loss observed at $6 \mathrm{M}$ compared to $2 \mathrm{M}$ for lbruR3 and $30 \%$

169 loss between IbruR1 and IbruR1 +3M (Fig. 3A). To further characterise these evolutions, we

170 performed a correlation study using REMI analysis first looking at this peak evolution compared to IbruR3 before treatment (IbruR3 BT) and then versus IbruR3 2M. As observed previously, the fluctuation in peak content at $2 \mathrm{M}$ did not exceed $20 \%$ of what is observed before treatment, with the number of bivalent peaks (EZH2, SUZ12) going down and LSCE peaks going up and this fraction being enriched for cis-elements containing functional binding sites for SOX2 and PRDM1 (Fig. 3B). Overall, the score evolution could be separated into 4 subsets, (1) LSCE, (2) bivalent, (3) PRDM1/SOX2 and (4) the other transcription factors (TFs) of the ReMap database (Fig. 3C-I). In all samples analysed including IbruR3 2M, bivalent peaks were down and SOX2/PRDM1 high suggesting that it could be a characteristic of ibrutinib-treated CLL cells (Fig. 3C-I). Furthermore, we identified several types of CLL cell populations based on the evolution of both LSCE and the rest of the TFs. First, as expected for the three subsets showing a reduction of the total $\mathrm{H} 3 \mathrm{~K} 4 \mathrm{me} 3$ peak number, $6 \mathrm{M}, 9 \mathrm{M}$, and IbruR1 +3M (Fig. 3A), an increased proportion of all the other TF-associated peaks (subset 4) was observed, suggesting a loss of non-promoter cis elements (small) and a proportional enrichment for promoters (larger cis-elements containing more TF-binding sites) (Fig. 3C, D and $\mathrm{G})$. In addition, the number of $\mathrm{H} 3 \mathrm{~K} 4 \mathrm{me} 3 \mathrm{LSCE}$-associated peaks could be separated into (1) high, between $10 \%$ and $30 \%$ increase for lbruR1 and (2) low, less than $20 \%$ increase for the other samples (Fig. 3C, D and G). Furthermore, IbruR1 peak score was highly correlated with a sample previously characterised as activated CLL-cells $\left(T N \_7 d, R^{2}=0.95\right)$. These cells came from a front-line donor after 7 days on ibrutinib and characterised by a sharp increase 
190 in CLL cell count in the PB in the first days of treatment (17) (Fig. 3I). Therefore, by merging

191 these two observations combined with the global level of H3K27me3, we could separate these

192 samples into four distinct groups; newly generated activated CLL cells (IbruR1, TN_7d), early

193 ( $2 \mathrm{M}$ and $33 \mathrm{M}$, the correlation between both peak populations being $\mathrm{R}^{2}=0.67$ ), intermediate

194 (9M) and late/oldest (6M, IbruR1 +3M) quiescent-like cells (Fig. 3C-I).

The difference in $\mathrm{H} 3 \mathrm{~K} 4 \mathrm{me} 3$ peak intensity is indicative of variations in transcriptional activity in these cells different phenotypes, from activated CLL cells and progressing toward different degrees of quiescence with a progressive disappearance of $\mathrm{H} 3 \mathrm{~K} 27 \mathrm{ac}$ genome-wide and $\mathrm{H} 3 \mathrm{~K} 4 \mathrm{me} 3$ at LSCE-associated peaks, the oldest cells also losing H3K27me3.

First, to confirm that our classification of the different subcategories of cis-elements containing identified TFs was correct, we took advantage of a new study which classified each open chromatin region in CLL into 12 chromatin states depending of their content in specific histone marks (20). Each H3K4me3 peak identified in our analysis was marked in red if they were present in a specific chromatin state and in white if not. Finally, we ranked these peaks for each analysed sample based on fold change between this sample and IbruR3 BT (Fig. 4AG). The same analysis was performed with 60 selected TFs from the ReMap database

209 (Supplementary Fig. S3A). By comparing both analyses, we confirmed that EZH2 and SUZ12

210 were enriched in the decreased fraction of IbruR1, which were enriched for the chromatin

211 states E5, E6, E7 representing polycomb-dependent silenced and bivalent cis-elements.

212 Similarly, H3K4me3 peaks associated with lineage specific transcription factors including

213 SOX2, PRDM1, BCL6 and BATF were enriched in the increased fraction, which contain E1,

214 E8 and E9 non-promoter cis-elements (Fig. 4, Supplementary Fig. S3A). Moreover, the number of peaks in this increased fraction for each CLL sample was correlated with the level 216 of activation of these cells (Figs. 3 and 4). 
To fine tune the analysis of the changes of peak population in these different samples,

218 we separated these H3K4me3 peaks in 4 subsets depending on fold change versus IbruR3

219 BT and performed the same analysis as described in Fig. 3 (Supplementary Fig. S3B). Overall,

220 the peak content for the different subsets was highly correlated between all samples with few

221 noticeable variations (Supplementary Fig. S3C-F). As expected, bivalent regions (SUZ12,

222 EZH2) were mainly found in the decreased subset, with IbruR1 +3M being the only sample showing a reduced number of these elements compensated by an accumulation of SUZ12associated cis-element in the increased subset (Supplementary Fig. S3C and S3E). Moreover, further loss of bivalent cis-elements with time on ibrutinib and the reciprocal gain of SOX2associated regions were observable for the stable and increased subsets when comparing samples collected after 6 months treatment and IbruR3 2M (Supplementary Fig. S3C and D). The most important variations could be seen when analysing the peak content of the increased subset, probably due to the small number of peaks in it (Supplementary Fig. S3C). Notably,

230 PRDM1-containing validated binding sites were enriched at $6 \mathrm{M}, 9 \mathrm{M}$ and for IbruR1 and 6M, 9M, 33M and IbruR1 +3M were associated with a decrease in TAp73A-containing ciselements (Supplementary Fig. S3C). In contrast at 33M, the increased subset was enriched

233 for cis-elements containing validated ONECUT1- and MEIS1-binding sites and depleted from 234 BCL6 sites suggesting a possible change in the TF network regulating proliferation after disease progression for this patient (Supplementary Fig. S3C). Finally, 6M and IbruR1 were the two samples showing no correlation when compared to IbruR3 $2 \mathrm{M}$ in the absent subset, with the observed peak scores being indicative of an important loss of $\mathrm{H} 3 \mathrm{~K} 4 \mathrm{me} 3$ at promoters

238 (large regions targeted by multiple TFs) (Supplementary Fig. S3F). At 6M, it could be explained by the loss of almost 3000 peaks compared to $33 \mathrm{M}$. In contrast, further analysis revealed that, for IbruR1, it was dependent upon promoter reorganisation and nucleosome loss as seen for selected samples (Supplementary Fig. S4A). Therefore, activated-CLL cells were characterised by an accumulation of $\mathrm{H} 3 \mathrm{~K} 4 \mathrm{me} 3$ at non-promoter elements and by chromatin reorganisation leading to nucleosome loss at active promoters. 
To conclude this analysis of $\mathrm{H} 3 \mathrm{~K} 4 \mathrm{me} 3$ quantitative changes, we also compared the evolution of the intensity of these peaks with time to determine if these changes were random

246 or conserved between samples (Fig. 5A). We determined that $57 \%$ of the enriched peaks seen

247 at $9 \mathrm{M}$ are also enriched at $6 \mathrm{M}$ and $33 \mathrm{M}$. Similarly, $81 \%$ of the decreased peaks at $33 \mathrm{M}$ are 248 also decreased at $6 \mathrm{M}$ and $9 \mathrm{M}$ suggesting a significant level of stability between untreated and on ibrutinib treated samples (Fig. $5 \mathrm{~A}$ ). Moreover, $98 \%$ of the peaks higher in IbruR $1+3 \mathrm{M}$ than in IbruR3 BT were also higher three months before (Fig. 5A). We have associated those ciselements conserved in $6 \mathrm{M}, 9 \mathrm{M}$ and $33 \mathrm{M}$ with their closest genes and determined functional associations between these genes by gene ontology analysis (Supplementary Fig. S4A, S5A, B and table 4). However, the meaning of these changes is unclear because it occurred in the context of a global decrease in $\mathrm{H} 3 \mathrm{~K} 27 \mathrm{ac}$ and therefore reduced transcriptional activity. Similarly, focusing on the $98 \%$ of peak high in both IbruR1 samples, non-canonical, positive regulation of differentiation and actin cytoskeleton organisation were among the function enriched for IbruR1 compared to IbruR3 BT (Supplementary Fig. S4A, Supplementary table 4). Interestingly, genes associated with actin cytoskeleton organisation (PROX1, ACTN1, ATR, COBLL1) were found to be enriched before treatment compared to $6 \mathrm{M}-33 \mathrm{M}$ and reduced compared to IbruR1 (Supplementary Fig. S4B). This revealed alternative H3K4me3 peaks in regions corresponding to potential alternative promoters for IbruR1 suggesting a possible aberrant regulation of these genes in CLL cells for this first relapse patient (Supplementary Fig. S5B). To conclude, gene ontology analysis comparing genes closest to peaks enriched in IbruR1 $+3 \mathrm{M}$ compared to IbruR3 at $6 \mathrm{M}, 9 \mathrm{M}$ and $33 \mathrm{M}$ identified several pathways specifically activated in quiescent-like IbruR1 including JNK, non-canonical WNT and pro-inflammatory signalling pathways (Supplementary Fig. S5B). JNK signalling pathway has been shown to mediate apoptosis in response to various treatment in CLL (21-23), but is inactivated by NF$\kappa B(24)$. 
To identify chromatin structure specifically associated with IbruR1 and considering the

273 fact that this first CLL sample was showing characteristics of activated-CLL cells, we

274 compared H3K4me3 for this patient with 5 previously analysed patients for whom we had

275 chromatin at a time point corresponding to ibrutinib-dependent lymphocytosis (17). We

276 performed gene ontology analysis for the closest genes associated with H3K4me3 peaks

277 found 2.5-fold higher for IbruR1 compared to all 5 other samples. Only 15 genes were found

278 associated with specific functions, including regulation of growth and the non-canonical Wnt

279 signalling pathways (Fig. 5B and C). Of particular interest among these genes was WNT5A,

280 a gene previously associated with ibrutinib resistance (25), and the fibroblast growth factor 20

281 (FGF20), a ligand also associated with proliferation and developmental growth (Fig. 5C).

$282 \mathrm{H} 3 \mathrm{~K} 4 \mathrm{me} 3$ data confirmed that this mark was specifically high at WNT5A promoter for both

283 IbruR1 samples and that a cis-element upstream of the FGF20 promoter was also specifically

284 enriched for this histone mark in IbruR1, but lower at +3M (Fig. 5D).

285 To determine if the changes observed in chromatin structure were supported by similar

286 changes in gene expression, we extracted mRNA from IbruR1, IbruR1 +3M, IbruR3 BT and

287 IbruR3 $33 \mathrm{M}$ as well as from an additional relapse patient IbruR2 and from 14 patients on

288 ibrutinib for which we collected CLL cells from before treatment and up to 18 months post

289 treatment initiation (Fig. 6A, Supplementary Fig S7 and S8). None of these later patients

290 showed any sign of disease progression at the time of the study. We compared gene expression for two proliferation markers (Ki67, PCNA), FGF20 and WNT5A. Among the samples from relapse patients, only lbruR1 expressed both proliferation markers reinforcing the view that these CLL cells were characteristic of activated cells and that IbruR2, IbruR3 $33 \mathrm{M}$ and IbruR1 $+3 \mathrm{M}$ were in a more resting/dormant stage (Fig. 6A).

Unexpectedly, PCNA and Ki67 were also expressed in patients 7, 8, 9, 13 and 14 at various time points (Fig. 6A). A more extensive gene expression analysis indicated a close correlation between expression of these proliferation markers and genes including $B C L 2$,

$298 E Z H 1 / 2$, SOX11, CD38, a surface protein for which enzymatic activity has been correlated 299 with enhanced growth and cellular mobility and poor risk in CLL (26), interferon and chemokine 
300 receptors (Supplementary Fig. S7 and S8). Similarly, expression of pro-inflammatory related

301 genes including TLR4, TLR9 and TNFA was also closely correlated, with the expression of 302 these genes overlapping partially with the proliferative-associated ones as seen when 303 comparing TNFA expression with SOX11 or PCNA (Supplementary Fig. S7 and S8, Fig. 6A).

304 Finally, the gene expression profile of IbruR1 +3M was unique showing specific expression of WNT5A and FGFR1 (Fig. 6A, Supplementary Fig. S8A, right part) and substantial variations compared to 3 months earlier (Supplementary Fig. S9). FGFR1 expression was observed for IbruR1 $+3 \mathrm{M}$ and its ligand FGF20 was expressed in IbruR1 with a gene expression profile closely correlated to VDR, RXRA, ELK1 and ILB1 (Supplementary Fig. S8A). These data indicated that expression of two potential drivers of resistance were only detected in CLL cells after a prolonged phase of quiescence (H3K27me3 low).

Discussion

CLL cells present significant phenotypic variations depending on their age and compartment that they come from (27). In response to ibrutinib, after an initial phase of lymphocytosis, the chromatin structure of CLL cells changes progressively from activated cells toward a quiescent-like phenotype losing both $\mathrm{H} 3 \mathrm{~K} 27 \mathrm{ac}$ and $\mathrm{H} 3 \mathrm{~K} 27$ me3 genome-wide as well as H3K4me3 primarily at bivalent cis-elements characteristic of resting CLL cells $(14,17)$. Here, we have shown an expected chromatin and gene expression profile of activated CLL cells in one patient on ibrutinib showing disease progression, whereas two other "relapse" samples have chromatin characteristics of quiescent-like cells. These data suggest that relapse is not a continuous process and as observed in the absence of treatment, that these cells could be either in a proliferating or a quiescent phase (Fig. 6B).

Interestingly, CLL cells from patients on ibrutinib display some similar chromatin characteristics, independent of disease progression. These include loss of H3K4me3 at

326 bivalent cis-elements and, reciprocally, gain of the same histone PTM at regions containing

327 validated SOX2-binding motifs. SOX2 is of interest as a key regulator of both the early 
328 development and maintenance of undifferentiated embryonic stem cells and has been

329 characterised as an oncogene in multiple cancers (28). EZH2-, SUZ12- and SOX2-validated

330 binding sites included into the ReMap database come from studies performed in Embryonic

331 Stem Cells (ESCs), including studies by the ENCODE consortium (29-32). Our results

332 suggest that in response to ibrutinib CLL cells switch on or off cis-elements which are activated

333 or repressed in ESCs respectively, linking a quiescence-like phenotype with increased

334 'stemness' as described in disseminating tumour cells $(33,34)$.

335 Furthermore, the maintenance of this signature after relapse suggests that disease

336 progression, at least in its initial phase, occurs while cells are still in some degree sensitive to

337 ibrutinib. For the C481S BTK mutant (IbruR3), these data agree with the fact that more than

$33850 \%$ of the cells do not carry the mutation in the initial phase of disease progression. This

339 discrepancy between disease progression and the percentage of BTK mutants in the growing

340 cell population is characteristic of ibrutinib treatment in CLL (12).

341 Moreover, our data suggest additional chromatin structure variations in quiescent-like

342 CLL cells, possibly dependent on two parameters - (i) disease progression and (ii) age of the

343 cells. EZH2 expression is restricted to proliferative cells in cancer and in non-transformed

344 human cells (35) and, accordingly, to proliferation centres in CLL and to the germinal centre

345 in B cells (36-38). Consequently, loss of H3K27me3 in quiescent-like CLL cells can be

346 interpreted as a prolonged exit of these cells from the cell cycle, characteristic of aging cells.

347 Here, two CLL samples amongst those investigated at greatest depth, IbruR3 6M and IbruR1

$348+3 \mathrm{M}$, share a profile of drastic reduction in $\mathrm{H} 3 \mathrm{~K} 27$ me3 suggesting that both have been

349 maintained in G0 phase for an extended period of time. Interestingly, these samples also show

350 common characteristics associated with quiescent-like cells including loss and gain of

351 H3K4me3 positive cis-elements associated with Tap73 and PRDM1, respectively. Tap73

352 promotes the Warburg effect and proliferation in cancer (39); therefore, silencing of Tap73-

353 associated cis-elements might be a characteristic of this quiescence. Similarly, PRDM1/Blimp-

354 1, a master regulator of B cell differentiation, has been shown to actively repress a number of 
355 functions in B cells including cell-cell adhesion (40), which we show to be associated with an

356 increase in $\mathrm{H} 3 \mathrm{~K} 4 \mathrm{me} 3$ at $6 \mathrm{M}, 9 \mathrm{M}$ and $33 \mathrm{M}$ compared to pre-treatment.

Whilst the IbruR1 $+3 \mathrm{M}$ epigenome is comparable to that of IbruR3 $6 \mathrm{M}$, suggesting a

358 prolonged exit from the cell cycle, the level of H3K27ac is significantly higher in these cells,

359 indicative of enhanced transcriptional activity matched by a gene expression profile which is

360 distinct from other quiescent-like cells. Additionally, these cells show an enrichment for

$361 \mathrm{H} 3 \mathrm{~K} 4 \mathrm{me} 3$ at bivalent regions, which could be due to the concomitant loss of the repressive

362 mark (H3K27me3) and an increase in global transcriptional activity. In these cells, WNT5A

363 and FGFR1, which have been described as potential candidates to mediate ibrutinib-

364 resistance, show 44 - and 17-fold higher expression compared to cells three months earlier,

365 which have an activated phenotype. WNT5A can enhance the proliferation and migration of

366 CLL cells via activation of the non-canonical WNT pathway $(25,41)$, a mechanism which is not inhibited by ibrutinib (42). Similarly, activation of FGFR targets four internal signalling pathways RAS-MAPK, PI3K-AKT, PLC $\gamma$, and STAT (43), suggesting that it might also induce proliferation in CLL cells by bypassing BTK inhibition. These pathways may act synergistically, with canonical WNT5A signalling increasing the transcription of FGF20, an FGFR1 ligand (44). Detecting transient expression of proliferation markers, including Ki67 and EZH2, in cells from four patients showing no clinical sign of disease progression reinforced the view that CLL cells are still able to enter cell cycle under ibrutinib-dependent repression of the BCR signalling pathway, as shown by analysis of IbruR3 9M epigenome. These data argue that CLL cells from patients treated with ibrutinib are still able to enter cell cycle in the absence of detectable disease progression.

\section{Conclusions}

We have shown that the chromatin structure of CLL cells from patients on ibrutinib changes dynamically for reasons independent of sensitivity to the treatment. These changes are coordinated and consistently correlated with the age of the cells and cannot be explained by random variations due to technical inconsistency. Accordingly, CLL cells from relapse 
383 patients can be either in a quiescent-like or proliferating state. In addition, our data suggest

384 that older cells are the ones which might be responding to signalling which is capable of 385 bypassing BTK inhibition. As mentioned in the introduction, these phenotype-associated 386 variations are relevant for the particular individuals included in this study and suggest that any 387 mechanistic analysis aimed at identifying the phenotypic specificity of relapsing cells would 388 have to take the inherent epigenomic variation into consideration.

\section{Methods}

Isolation of CLL Cells

Peripheral blood was collected from patients with informed written consent (St. James's University Hospital, approval number 14/YH/0034; IcICLLe EudraCT number: 2012003608-11). Peripheral blood mononuclear cells (PMBCs) were isolated by density gradient centrifugation using Lymphoprep ${ }^{\mathrm{TM}}$ (Axis-Shield). CLL cells were further purified using CD19+ MicroBeads (Miltenyi Biotec).

\section{BTK and PLC $\gamma 2$ Mutation Detection}

100 ng of patient DNA extracted from CD19+ selected peripheral blood was used for PCR. A 527 bp fragment encompassing the BTKCys481 region was amplified using the following primers, GACTGCTCTGATTCCCACCA and TACTCCTAGGTCAGCCCCTTC. Similarly, three regions of the PLCY2 gene were amplified, a $190 \mathrm{bp}$ fragment which encompassed the PLCY2S707 region using the primers ACCAGGATCTTGGCATGTCA and GGGTAGCGCAGTCTCATCTT, A 323 bp fragment encompassing the PLCY2L845 region using the primers, TCCCCATGGACGTATCTGGT and TGGCATTCAAGACAGGACCC and a

407212 bp fragment covering the PLCY2R665 region using the primers AGGTGTCACTGGTGCCATTA and AGGTGATGGCATAGGAGTC. The amplified products were isolated using the QIAquick Gel Extraction Kit (Qiagen, Valencia, CA) and then sent for

410 Sanger sequencing (Source Bioscience, Nottingham, UK). 


\section{Immunoblot Analysis} Immunoblot analysis was conducted as published previously (17). Primary antibodies were as follows: H3K4me3 (Millipore; 04-745), H3K27me3 (Millipore; 07-449) and H3K27ac (Millipore; 07-360) were used as primary antibodies and anti-Total H3 (Abcam; ab1791) was used as loading controls.

\section{Chromatin Immunoprecipitation (ChIP) and ChIP-seq}

ChIP was performed as previously described $(45,46)$ using Dynabeads protein $G$ 420 (Invitrogen) with $2.4 \mathrm{mg}$ per $10 \mathrm{ml}$ beads with $1 \mu \mathrm{g}$ anti-H3K4me3 (Millipore; 04-745), antiH3K27ac (Millipore; 07-360), anti-H3K27me3 (Millipore; 07-449). For sequencing (ChIP-seq), libraries were prepared using NEBNext ${ }^{\circledR}$ Ultra $^{\mathrm{TM}}$ II DNA Library Prep Kit (New England

Biolabs). See Supplementary Methods for sequencing run details.

\section{Bioinformatics Analysis}

See Supplementary Methods for further details. Sequence data from this study have been submitted to ArrayExpress (https://www.ebi.ac.uk/arrayexpress/) under accession number E-MTAB-8220.

Gene Expression Analysis

Total RNA was extracted from patient CLL samples using Direct-zol ${ }^{\mathrm{TM}}$ RNA MiniPrep

432 Kit (Zymo Research) and cDNA was prepare using M-MLV Reverse Transcriptase 433 (Invitrogen). Expression of target genes (Supplementary Table 2) was determined using 434 quantitative real-time polymerase chain reaction (qPCR) with GoTaq ${ }^{\circledR}$ qPCR Master Mix 435 (Promega) on a QuantStudio ${ }^{\mathrm{TM}} 7$ Flex Real-Time PCR System (Applied Biosystems). Relative expression $(\Delta \mathrm{Ct})$ was calculated from the $\mathrm{Ct}$ value of the average of 5 housekeeping genes

437 (PPP6C, GAPDH, RPL13A, $\beta$-Actin, B2M) - Ct value of the gene of interest. 
439 list of abbreviations

440

$441 \quad$ ACTN1 actinin alpha 1

442 BATF basic leucine zipper ATF-like transcription factor

443 BT before treatment

444 BTK bruton's tyrosine kinase

445 B2M beta 2 microglobulin

446 CDK8 cyclin dependent kinase 8

447 CLL chronic lymphocytic leukaemia

448 COBLL1 cordon-bleu WH2 repeat protein like 1

449 ESC embryonic stem cell

450 EZH2 enhancer of zeste 2 polycomb repressive complex 2

$451 \quad$ FGF20 fibroblast growth factor 20

452 FGFR1 fibroblast growth factor receptor 1

$453 \quad$ ILB1 interleukin 1 beta

454 GAPDH glyceraldehyde 3 phosphate dehydrogenase

455 GO gene ontology

456 IbruR ibrutinib resistant

457 JNK c-Jun N-terminal kinase

$458 \quad$ Ki67 marker of proliferation Ki-67

459 KLF4 kruppel like factor 4

460 LSCE lineage specific cis-elements

461 MAPK map kinase

462 MED12 mediator complex subunit 12

$463 \quad$ NF-kB nuclear factor kappa B

464 ONECUT1 one cut homeobox 1

465 PB peripheral blood 
466 PCNA proliferating cell nuclear antigen

467 PI3K phosphatidylinositol 3 kinase

$468 \quad$ PLC $\gamma 2$ phospholipase C gamma 2

469 PMBC peripheral blood mononuclear cell

$470 \quad$ PPP6C protein phosphatase 6 catalytic subunit

$471 \quad$ PRC2 polycomb receptor complex 2

472 PRDM1 PR/SET domain 1

473 PROX1 prospero homeobox 1

474 PTM post-translational modification

475 qPCR quantitative real-time polymerase chain reaction

476 RPL13A ribosomal protein L13a

477 REMI reMap and epigenetic mark intersection

478 RXRA retinoid X receptor alpha

479 SOX2 SRY-box transcription factor 2

480 SOX11 SRY-Box transcription factor 11

481 STAT signal transducer and activator of transcription

482 Tap73 transformation related protein 73

483 TGF $\beta$ transforming growth factor beta

484 TF transcription factor

485 TLR Ttoll like receptor

486 TN treatment naive

487 TNFA tumor necrosis factor

488 VDR vitamin D receptor

489 WNT5A wnt family member 5A

490

491 Declarations

492 
493 Ethical approval and consent to participate

494 Peripheral blood was collected from patients with informed written consent (St. James's

495 University Hospital, approval number 14/YH/0034; IcICLLe EudraCT number: 2012-003608496 11).

497

498 Consent for publication

499 Not applicable

500

501 Availability of supporting data

502 Sequence data from this study have been submitted to ArrayExpress

503 (https://www.ebi.ac.uk/arrayexpress/) under accession number E-MTAB-8220.

504

505 Competing interests

506 The authors declare no competing financial interests.

507

508 Funding

509 This work was supported by Bloodwise trial associated research project (14016).

510

511 Authors' contribution

$512 \mathrm{KH}, \mathrm{FT}, \mathrm{CE}$ and AR performed the experiments and generated figures. PL, IS analysed the

513 data and PL wrote the paper. $\mathrm{PH}$ was involved in the initial design of the project and

514 contributed to provide essential research material. DN participated to the data analysis. $\mathrm{KH}$,

515 IS, PH and DN participated to the redaction of the manuscript.

516

517 Acknowledgments

518 Not applicable

519

520 Authors' information 


\section{Affiliations}

522 Division of Haematology and Immunology, Leeds Institute of Medical Research at St.

523 James's, University of Leeds, Leeds, UK

524 Katie B. Holmes, Ildar I. Sadreev, Charlotte L. Evans, Fraser Thomas, Darren J. Newton ${ }^{1}$,

525 Peter Hillmen and Pascal F. Lefevre

526 Haematological Malignancy Diagnostic Service (HMDS), St. James's Institute of Oncology,

527 Bexley Wing, Beckett Street, Leeds LS9 7TF, UK

528 Andy C. Rawstron

529 Corresponding authors

530 Correspondence to Pascal F. Lefevre

532 References

533

534 1. Howlader N NA, Krapcho M, Garshell J, Neyman N, Altekruse SF, Kosary CL, Yu M, Ruhl 535 J, Tatalovich Z, Cho H, Mariotto A, Lewis DR, Chen HS, Feuer EJ, Cronin KA (eds). SEER Cancer 536 Statistics Review, 1975-2010. Bethesda, MD: National Cancer Institute. 2013.

537 2. Fabbri G, Dalla-Favera R. The molecular pathogenesis of chronic lymphocytic 538 leukaemia. Nature Reviews Cancer. 2016;16(3).

539 3. Byrd JC, Brown JR, O'Brien S, Barrientos JC, Kay NE, Reddy NM, et al. Ibrutinib versus

540 ofatumumab in previously treated chronic lymphoid leukemia. The New England journal of 541 medicine. 2014;371(3):213-23.

542 4. Burger JA, Tedeschi A, Barr PM, Robak T, Owen C, Ghia P, et al. Ibrutinib as Initial 543 Therapy for Patients with Chronic Lymphocytic Leukemia. The New England journal of 544 medicine. 2015;373(25):2425-37. 
545 5. Collett L, Howard DR, Munir T, McParland L, Oughton JB, Rawstron AC, et al.

546 Assessment of ibrutinib plus rituximab in front-line CLL (FLAIR trial): study protocol for a phase

547 III randomised controlled trial. Trials. 2017;18(1):387.

548 6. Hillmen P, Rawstron AC, Brock K, Munoz-Vicente S, Yates FJ, Bishop R, et al. Ibrutinib 549 Plus Venetoclax in Relapsed/Refractory Chronic Lymphocytic Leukemia: The CLARITY Study. J $550 \quad$ Clin Oncol. 2019:JCO1900894.

551 7. Kater AP, Levin MD, Niemann CU. Ibrutinib and Venetoclax for First-Line Treatment of

552 CLL. The New England journal of medicine. 2019;381(8):788-9.

553 8. Kaur V, Swami A. Ibrutinib in CLL: a focus on adverse events, resistance, and novel 554 approaches beyond ibrutinib. Annals of hematology. 2017.

555 9. Ahn IE, Underbayev C, Albitar A, Herman SE, Tian X, Maric I, et al. Clonal evolution 556 leading to ibrutinib resistance in chronic lymphocytic leukemia. Blood. 2017;129(11):1469-79.

557 10. Jain $\mathrm{P}$, Thompson $\mathrm{PA}$, Keating $\mathrm{M}$, Estrov Z, Ferrajoli $\mathrm{A}$, Jain $\mathrm{N}$, et al. Long-term 558 outcomes for patients with chronic lymphocytic leukemia who discontinue ibrutinib. Cancer. 2017;123(12):2268-73.

560 11. Herman SE, Mustafa RZ, Gyamfi JA, Pittaluga S, Chang S, Chang B, et al. Ibrutinib 561 inhibits BCR and NF-kappaB signaling and reduces tumor proliferation in tissue-resident cells 562 of patients with CLL. Blood. 2014;123(21):3286-95.

563 12. Landau DA, Sun C, Rosebrock D, Herman SEM, Fein J, Sivina M, et al. The evolutionary

564 landscape of chronic lymphocytic leukemia treated with ibrutinib targeted therapy. Nature 565 Communications. 2017;8(1):2185.

566 13. Schmidl C, Vladimer GI, Rendeiro AFF, Schnabl S, Krausgruber T, Taubert C, et al. 567 Combined chemosensitivity and chromatin profiling prioritizes drug combinations in CLL. 568 Nature chemical biology. 2019;15(3):232-40. 
570 mapping and single-cell immune profiling define the temporal dynamics of ibrutinib drug

571 response in chronic lymphocytic leukemia. bioRxiv. 2019:597005.

572 15. de Rooij MF, Kuil A, Geest CR, Eldering E, Chang BY, Buggy JJ, et al. The clinically active

573 BTK inhibitor PCl-32765 targets B-cell receptor- and chemokine-controlled adhesion and

574 migration in chronic lymphocytic leukemia. Blood. 2012;119(11):2590-4.

575 16. Pastore A, Gaiti F, Lu SX, Brand RM, Kulm S, Chaligne R, et al. Corrupted coordination

576 of epigenetic modifications leads to diverging chromatin states and transcriptional

577 heterogeneity in CLL. Nature Communications. 2019;10(1):1874.

578 17. Holmes KB, Sadreev, II, Rawstron AC, Munir T, Westhead DR, Hillmen P, et al. Ibrutinib

579 induces chromatin reorganisation of chronic lymphocytic leukaemia cells. Oncogenesis. $580 \quad 2019 ; 8(5): 32$.

581 18. Woyach JA, Furman RR, Liu T-MM, Ozer HG, Zapatka M, Ruppert AS, et al. Resistance 582 Mechanisms for the Bruton's Tyrosine Kinase Inhibitor Ibrutinib. The New England journal of 583 medicine. 2014.

584 19. Furman RR, Cheng S, Lu P, Setty M, Perez AR, Guo A, et al. Ibrutinib Resistance in 585 Chronic Lymphocytic Leukemia. The New England journal of medicine. 2014.

586 20. Mallm JP, Iskar M, Ishaque N, Klett LC, Kugler SJ, Muino JM, et al. Linking aberrant 587 chromatin features in chronic lymphocytic leukemia to transcription factor networks. Mol 588 Syst Biol. 2019;15(5):e8339.

589 21. Peng K, Pan Y, Li J, Khan Z, Fan M, Yin H, et al. 11beta-Hydroxysteroid Dehydrogenase 590 Type 1(11beta-HSD1) mediates insulin resistance through JNK activation in adipocytes. Sci Rep. 2016;6:37160. 
22. Bates D, Feris EJ, Danilov AV, Eastman A. Rapid induction of apoptosis in chronic

593 lymphocytic leukemia cells by the microtubule disrupting agent BNC105. Cancer biology \& 594 therapy. 2016;17(3):291-9.

595 23. Paiva C, Godbersen JC, Soderquist RS, Rowland T, Kilmarx S, Spurgeon SE, et al. Cyclin596 Dependent Kinase Inhibitor P1446A Induces Apoptosis in a JNK/p38 MAPK-Dependent 597 Manner in Chronic Lymphocytic Leukemia B-Cells. PLoS One. 2015;10(11):e0143685.

598 24. Bubici C, Papa S, Pham CG, Zazzeroni F, Franzoso G. NF-kappaB and JNK: an intricate 599 affair. Cell Cycle. 2004;3(12):1524-9.

600 25. Yu J, Chen L, Cui B, Wu C, Choi MY, Chen Y, et al. Cirmtuzumab inhibits Wnt5a-induced 601 Rac1 activation in chronic lymphocytic leukemia treated with ibrutinib. Leukemia. $602 \quad 2017 ; 31(6): 1333$.

603 26. Vaisitti T, Audrito V, Serra S, Buonincontri R, Sociali G, Mannino E, et al. The enzymatic 604 activities of CD38 enhance CLL growth and trafficking: implications for therapeutic targeting. 605 Leukemia. 2015;29(2):356-68.

606 27. Herndon TM, Chen SS, Saba NS, Valdez J, Emson C, Gatmaitan M, et al. Direct in vivo 607 evidence for increased proliferation of CLL cells in lymph nodes compared to bone marrow 608 and peripheral blood. Leukemia. 2017;31(6):1340-7.

609 28. Wuebben EL, Rizzino A. The dark side of SOX2: cancer - a comprehensive overview. $610 \quad$ Oncotarget. 2017;8(27):44917-43.

611 29. Lister R, Pelizzola M, Dowen RH, Hawkins RD, Hon G, Tonti-Filippini J, et al. Human 612 DNA methylomes at base resolution show widespread epigenomic differences. Nature. $6132009 ; 462(7271): 315-22$.

614 30. Soufi A, Donahue G, Zaret KS. Facilitators and impediments of the pluripotency 615 reprogramming factors' initial engagement with the genome. Cell. 2012;151(5):994-1004. 
616 31. Xu K, Wu ZJ, Groner AC, He HH, Cai C, Lis RT, et al. EZH2 oncogenic activity in

617 castration-resistant prostate cancer cells is Polycomb-independent. Science. $618 \quad 2012 ; 338(6113): 1465-9$.

619 32. Abayakoon P, Lingford JP, Jin Y, Bengt C, Davies GJ, Yao S, et al. Discovery and 620 characterization of a sulfoquinovose mutarotase using kinetic analysis at equilibrium by 621 exchange spectroscopy. The Biochemical journal. 2018;475(7):1371-83.

622 33. Gay LJ, Malanchi I. The sleeping ugly: Tumour microenvironment's act to make or 623 break the spell of dormancy. Biochim Biophys Acta Rev Cancer. 2017;1868(1):231-8.

624 34. Weidenfeld K, Barkan D. EMT and Stemness in Tumor Dormancy and Outgrowth: Are 625 They Intertwined Processes? Front Oncol. 2018;8:381.

626 35. Bracken AP, Pasini D, Capra M, Prosperini E, Colli E, Helin K. EZH2 is downstream of 627 the pRB-E2F pathway, essential for proliferation and amplified in cancer. The EMBO Journal. $628 \quad 2003 ; 22(20): 5323-35$.

629 36. van Galen JC, Dukers DF, Giroth C, Sewalt RG, Otte AP, Meijer CJ, et al. Distinct 630 expression patterns of polycomb oncoproteins and their binding partners during the germinal 631 center reaction. European journal of immunology. 2004;34(7):1870-81.

632 37. Velichutina I, Shaknovich R, Geng H, Blood J-NA. EZH2-mediated epigenetic silencing 633 in germinal center B cells contributes to proliferation and lymphomagenesis. Blood. 2010.

634 38. Szurian K, Csala I, Marosvari D, Rajnai H, Dezso K, Bodor C, et al. EZH2 is upregulated 635 in the proliferation centers of CLL/SLL lymph nodes. Exp Mol Pathol. 2018;105(2):161-5.

636 39. Li L, Li L, Li W, Chen T, Bin Z, Zhao L, et al. TAp73-induced phosphofructokinase-1 637 transcription promotes the Warburg effect and enhances cell proliferation. Nat Commun. $638 \quad 2018 ; 9(1): 4683$. 
640 role of the transcription factor Blimp-1 in coordinating plasma cell differentiation. Nat 641 Immunol. 2016;17(3):331-43.

642 41. Yu J, Chen L, Cui B, Widhopf GF, 2nd, Shen Z, Wu R, et al. Wnt5a induces ROR1/ROR2 643 heterooligomerization to enhance leukemia chemotaxis and proliferation. J Clin Invest. $644 \quad 2016 ; 126(2): 585-98$.

645 42. Yu J, Chen Y, Chen L, Zhang L, Rassenti LZ, Widhopf GF, et al. Cirmtuzumab inhibits 646 ibrutinib-resistant, Wnt5a-induced Rac1 activation and proliferation in mantle cell lymphoma.

647 Oncotarget. 2018;9(37):24731-6.

648 43. Ornitz DM, Itoh N. The Fibroblast Growth Factor signaling pathway. Wiley 649 Interdisciplinary Reviews: Developmental Biology. 2015;4(3).

650 44. Katoh M, Katoh M. STAT3-induced WNT5A signaling loop in embryonic stem cells, 651 adult normal tissues, chronic persistent inflammation, rheumatoid arthritis and cancer 652 (Review). Int J Mol Med. 2007;19(2):273-8.

653 45. Lefevre P, Melnik S, Wilson N, Riggs AD, Bonifer C. Developmentally regulated 654 recruitment of transcription factors and chromatin modification activities to chicken lysozyme 655 cis-regulatory elements in vivo. Mol Cell Biol. 2003;23(12):4386-400.

656 46. Lefevre P, Witham J, Lacroix CE, Cockerill PN, Bonifer C. The LPS-induced 657 transcriptional upregulation of the chicken lysozyme locus involves CTCF eviction and 658 noncoding RNA transcription. Mol Cell. 2008;32(1):129-39. 
662 Fig. 1: Overview of chromatin structure changes with time on ibrutinib. (A) Identification 663 of the BTK C481S mutation by PCR after 32 months of ibrutinib treatment. The black arrows 664 indicate the position of the cysteine codon (UGC) mutated into serine (UCC) (B) Summary of 665 the ChIP-seq data focusing on two regions (chr18:27mb-37mb and Chr11:91,000-98,000) 666 representative of the obtained data and including a gene rich region surrounded by two gene 667 poor regions for which H3K27m3 enrichment is at background level. ChIP-seq has been performed for H3K27me3, H3K4me3 and H3K27ac looking at two patients (1) IbruR3 after 6, 9 and 33 months treatment and (2) IbruR1 at the time of disease progression detection (a) and 3 months later $(+3 \mathrm{M})$.

672 Fig. 2: Global level of H3K27ac does not follow disease progression. (A) Differential $\mathrm{H} 3 \mathrm{~K} 27 \mathrm{ac}$, as presented in Fig. 1 and defined as $\log _{2}\left(\mathrm{H} 3 \mathrm{~K} 27 \mathrm{ac}\right.$ fold-change) and $\log _{10}(-\mathrm{p}-$ value) between IbruR3 before treatment and at 6, 9 and 33 months as well as between IbruR1 and IbruR1 +3 months. Each H3K27ac peak was associated with its closest gene. (B) Same as A, but comparing IbruR3 at 6 and 9 months. (C) REMI analysis, cis-elements enriched for the H3K27ac mark were screened for the presence of validated binding sites for transcription factors and co-regulators (ReMap database). For each protein of the database, data were expressed as a score (equation (2) in Supplementary methods) corresponding to the number of peaks associated with a specific TF at 9M versus 6M. (D) Same as D but comparing IbruR3 BT and IbruR1.

682

683 Fig. 3: IbruR1 is presenting a H3K4me3 profile of activated-CLL cells. (A) Total H3K4me3 684 peak number between the different analysed subsets including IbruR3 BT, IbruR3 2M and TN 7d from a previous study (17). (B) REMI analysis, score variation for IbruR3 2M compared to before treatment. (C-I) Scatter diagrams representing a comparison between REMI scores for the different analysed samples (y axes) and reference profile (C-E, $\mathbf{G}$ and $\mathbf{H}$ ) IbruR3 2M, (F) IbruR3 6M and (I) TN 7d (x axes). 
690 Fig. 4: Changes in chromatin structure depending of the chromatin state. (A-G) Bar

691 coding presentation: chromatin states as defined previously (20) (E1 and E9,

692 H3K4me1/H3K27ac; E8, H3K4me1; E10, H3K4me3; E11, H3K27ac/H3K9ac; E5 and E6,

693 H3K27me3; E7, H3K27me3/H3K4me1/3) have been attributed a colour code depending of

694 their overlap with identified H3K4me3 peaks in IbruR1 and/or IbruR3 samples (red = present,

695 white $=$ absent). H3K4me3 peaks have been ranked based on fold-change between $(\mathbf{A})$ TN

696 7d, (B) IbruR3 2M, (C) IbruR3 6M, (D) IbruR3 9M, (E) IbruR3 33M, (F) IbruR1 and (G) IbruR1

$697+3 \mathrm{M}$ compared to IbruR3 BT.

698

699 Fig. 5: H3K4me3 peak intensity variation showed a significant degree of time dependent conservation. (A) Differential H3K4me3, as presented in Fig. 1 and defined as $\log _{2}(\mathrm{H} 3 \mathrm{~K} 4 \mathrm{me} 3$ fold-change) and $\log _{10}(-p$-value) between IbruR3 before treatment and at 6, 9, 33 months and IbruR1 as well as between IbruR1 and IbruR1 +3 months. Each H3K4me3 peak was associated with its closest gene. The percentage of peaks conserved at all time points $6 \mathrm{M}$, $9 \mathrm{M}$ and $33 \mathrm{M}$ are indicated for the decreased (red rectangle) and increased (green rectangle) fractions. Similarly, the percentage of peaks conserved in both IbruR1 and IbruR1 $+3 \mathrm{M}$ are indicated for the increased (grey rectangle) fractions. (B-C) Biological processes enriched at genes associated with differentially regulated H3K4me3 peaks (IbruR1 compared to 5 CLL samples from patients which received up to 7 days ibrutinib treatment (17); hypergeometric test $\mathrm{BH}-\mathrm{FDR}<0.05$ ). (D) Example of differential H3K4me3 peak intensity between the analysed samples (WNT5A and FGF20).

Fig. 6: WNT5A is specifically expressed in IbruR1. (A) gene expression analysis of CLL cells from PB for 14 patients collected before treatment or at 6, 9, 12 and 18M (as indicated on the Figure) compared to IbruR1 (R1), IbruR2 (R2) and IbruR (R3). PCNA, Ki67, FGF20 and WNT5A. Data are expressed relative to an average of 5 different control genes

716 Simplified model highlighting different level of transcriptional activity in CLL cells on ibrutinib. 
Figures

A

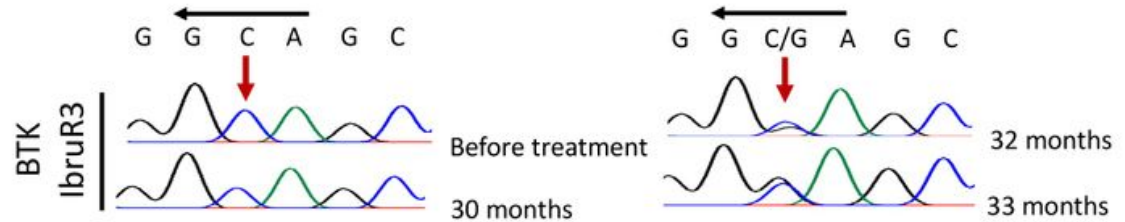

B

Chr18: $27 \mathrm{mb}-37 \mathrm{mb}$

C Chr11: 91,000-98,000

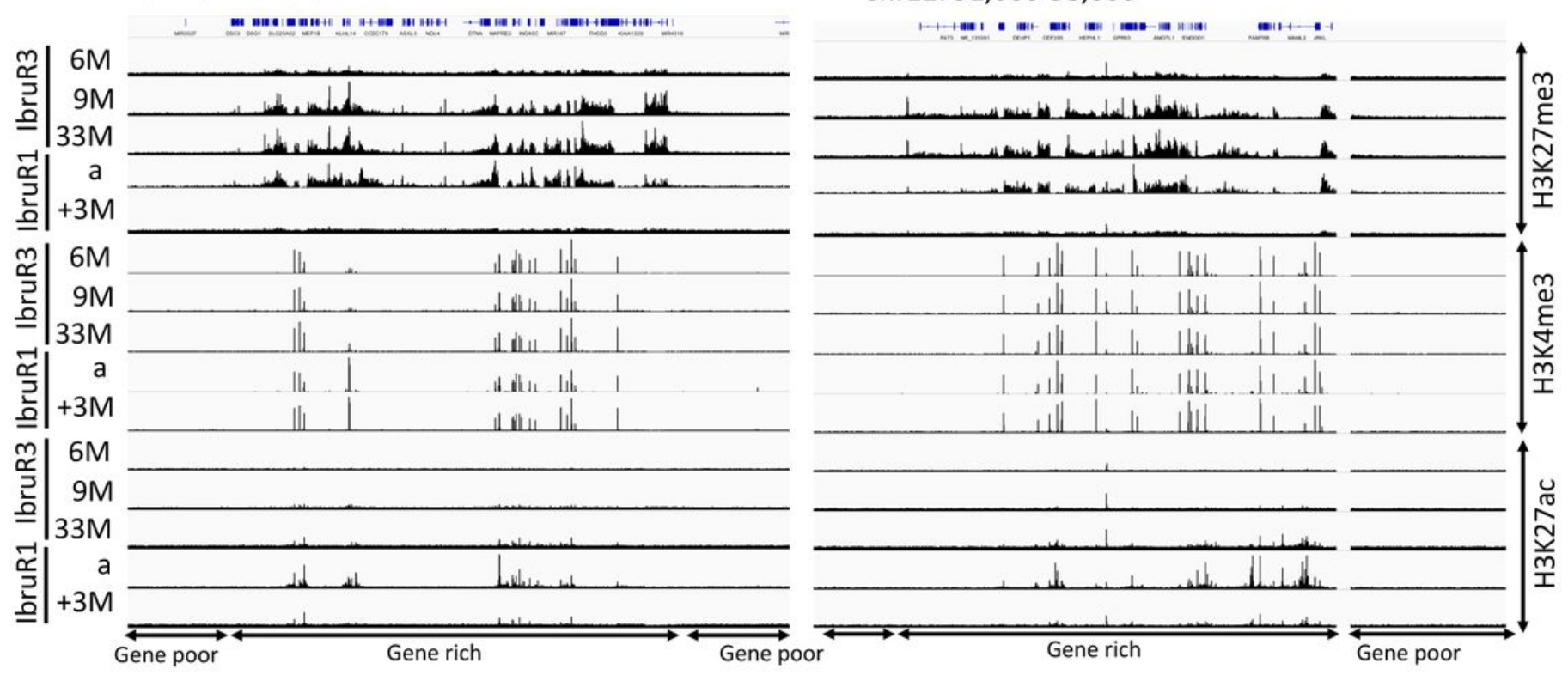

Figure 1

Overview of chromatin structure changes with time on ibrutinib. (A) Identification of the BTK C481S mutation by PCR after 32 months of ibrutinib treatment. The black arrows indicate the position of the cysteine codon (UGC) mutated into serine (UCC) (B) Summary of the ChIP-seq data focusing on two regions (chr18:27mb-37mb and Chr11:91,000-98,000) representative of the obtained data and including a gene rich region surrounded by two gene poor regions for which H3K27m3 enrichment is at background level. ChIP-seq has been performed for H3K27me3, H3K4me3 and H3K27ac looking at two patients (1) IbruR3 after 6, 9 and 33 months treatment and (2) IbruR1 at the time of disease progression detection (a) and 3 months later $(+3 M)$. 
A

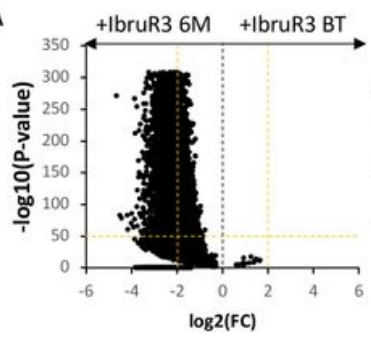

B

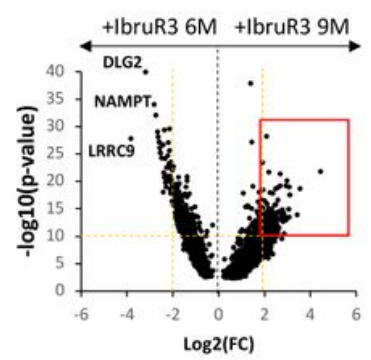

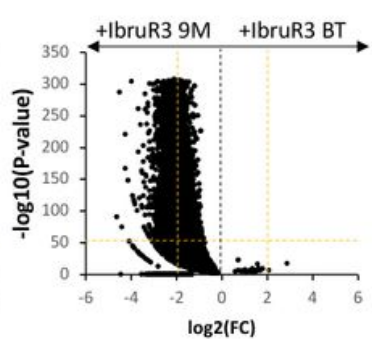

C

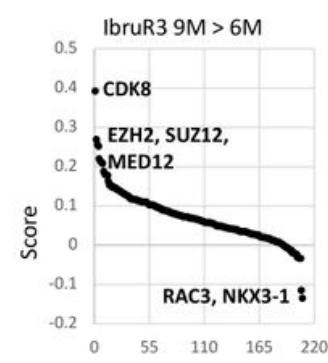

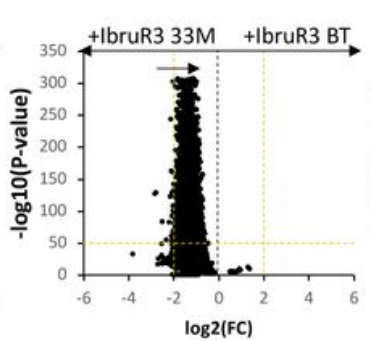

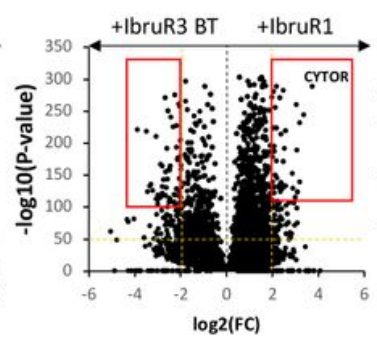

D

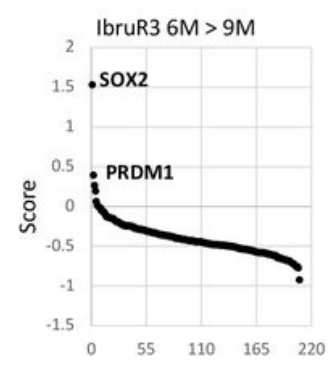

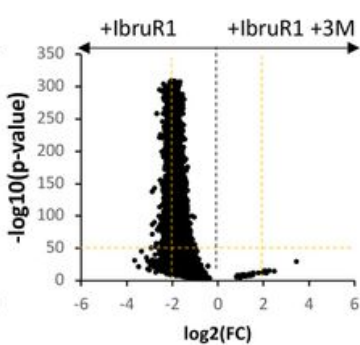
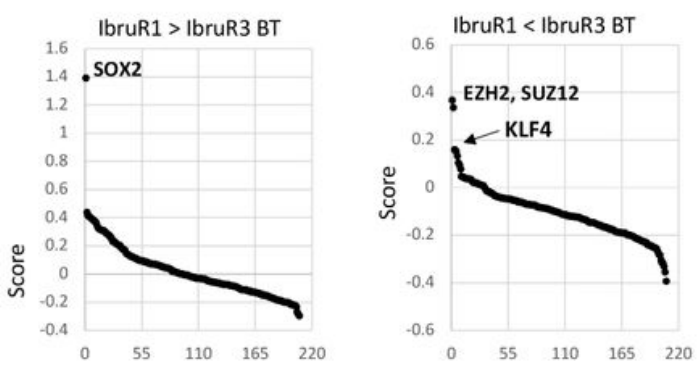

Figure 2

Global level of H3K27ac does not follow disease progression. (A) Differential H3K27ac, as presented in Fig. 1 and defined as log2(H3K27ac fold-change) and log10(-p-value) between IbruR3 before treatment and at 6, 9 and 33 months as well as between IbruR1 and IbruR1 +3 months. Each H3K27ac peak was associated with its closest gene. (B) Same as A, but comparing lbruR3 at 6 and 9 months. (C) REMI analysis, cis-elements enriched for the H3K27ac mark were screened for the presence of validated binding sites for transcription factors and co-regulators (ReMap database). For each protein of the database, data were expressed as a score (equation (2) in Supplementary methods) corresponding to the number of peaks associated with a specific TF at 9M versus 6M. (D) Same as D but comparing IbruR3 BT and IbruR1. 
A

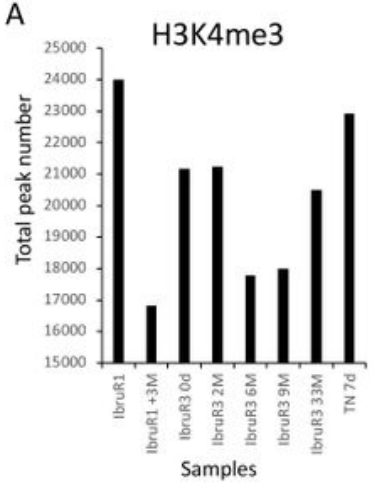

D

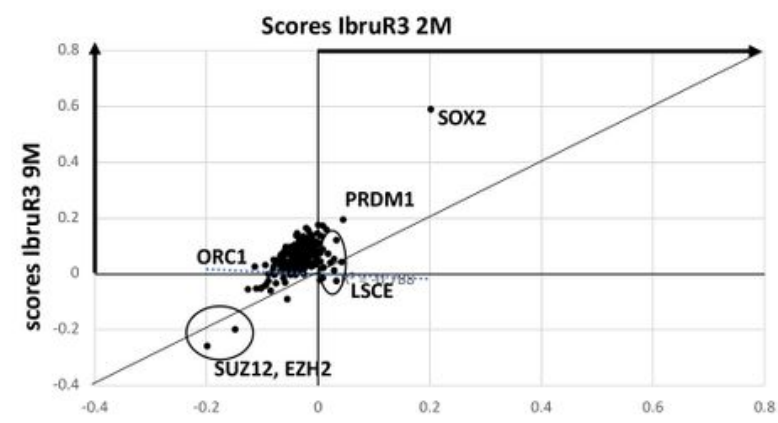

$\mathrm{F}$

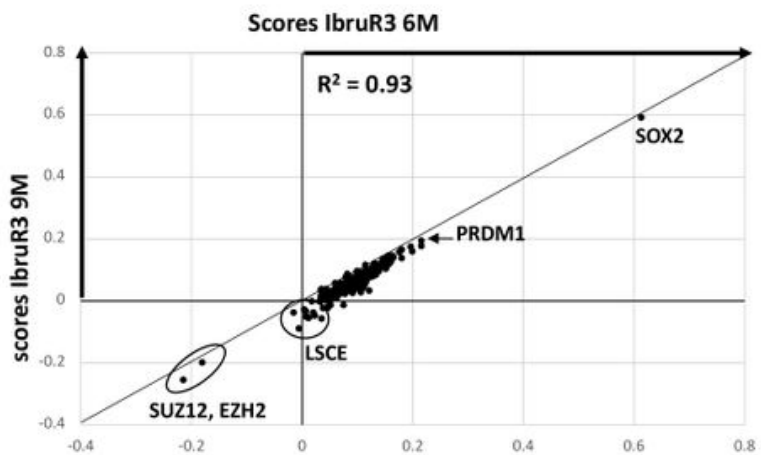

$\mathrm{H}$

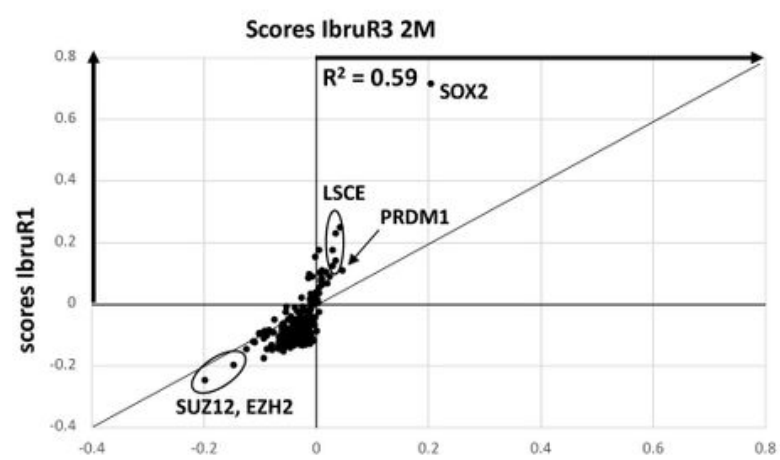

B

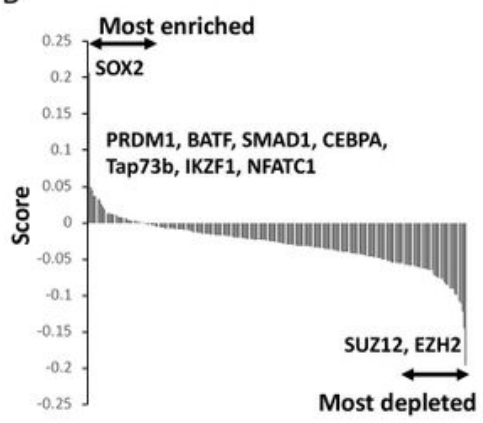

C
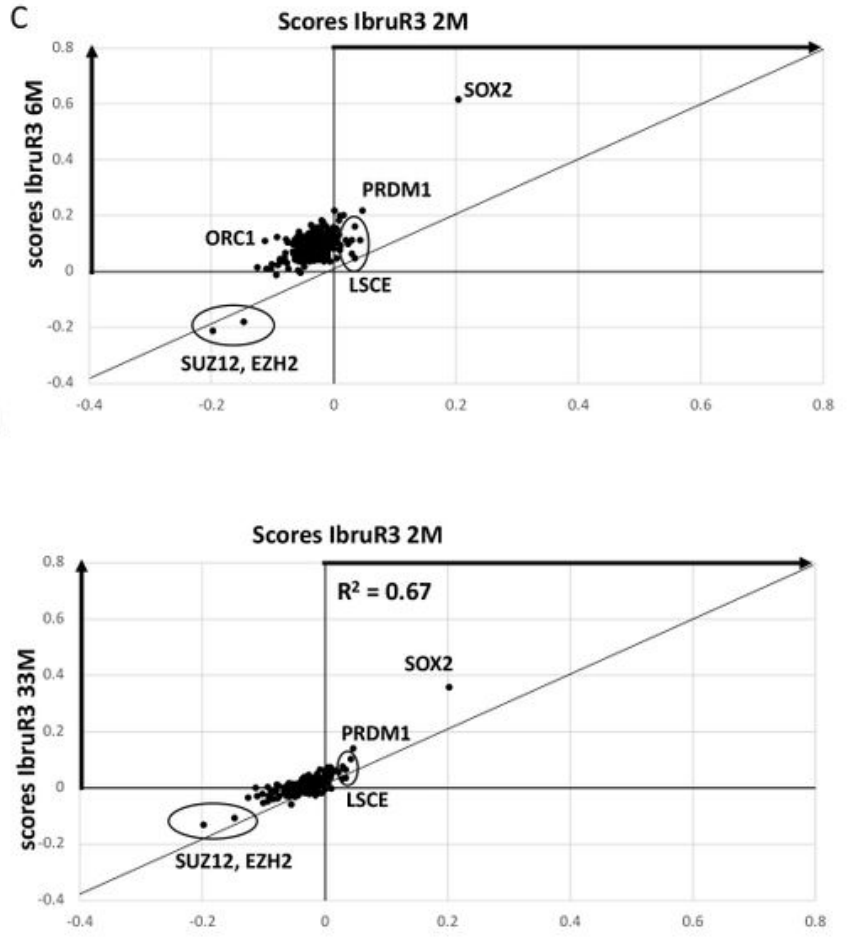

G

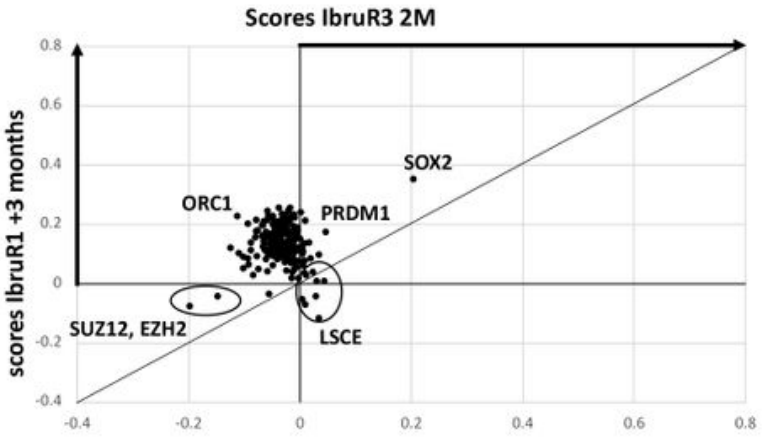

Scores TN 7d

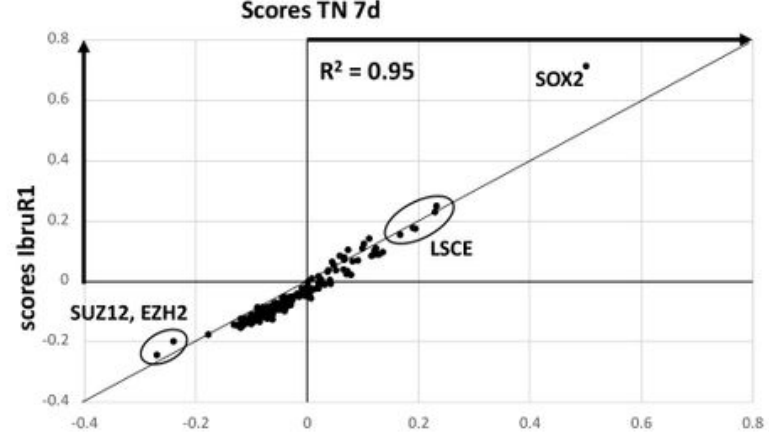

Figure 3

IbruR1 is presenting a H3K4me3 profile of activated-CLL cells. (A) Total H3K4me3 peak number between the different analysed subsets including IbruR3 BT, IbruR3 2M and TN 7d from a previous study (17). (B) REMI analysis, score variation for IbruR3 2M compared to before treatment. (C-I) Scatter diagrams representing a comparison between REMI scores for the different analysed samples (y axes) and reference profile (C-E, G and H) IbruR3 2M, (F) IbruR3 6M and (I) TN 7d (x axes). 
A

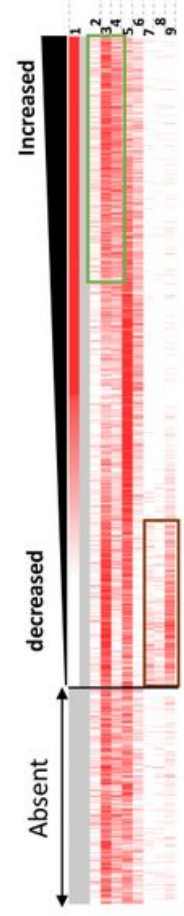

TN 7d
B

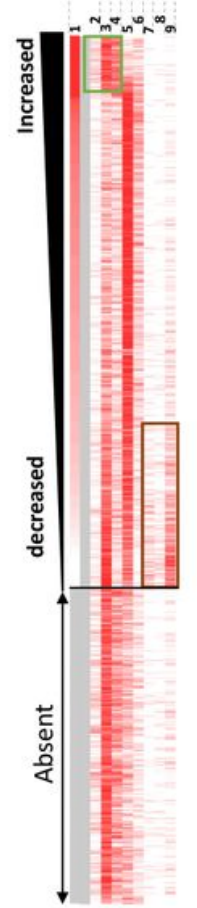

IbruR3 2M
C

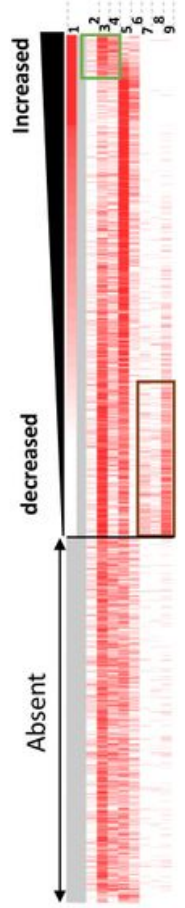

IbruR3 6M
D

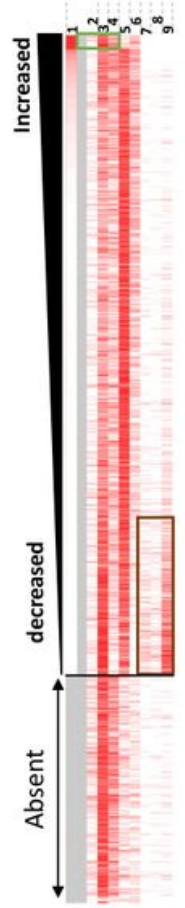

IbruR3 9M
E

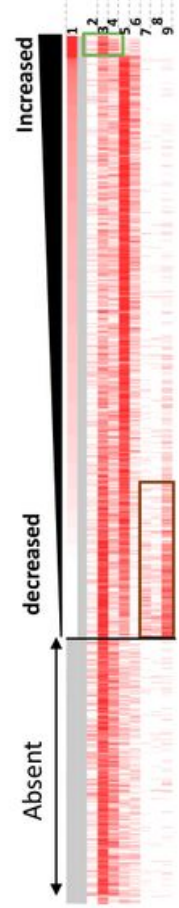

IbruR3 33M
F

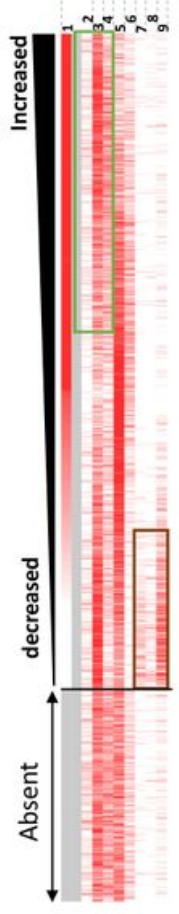

IbruR1
G

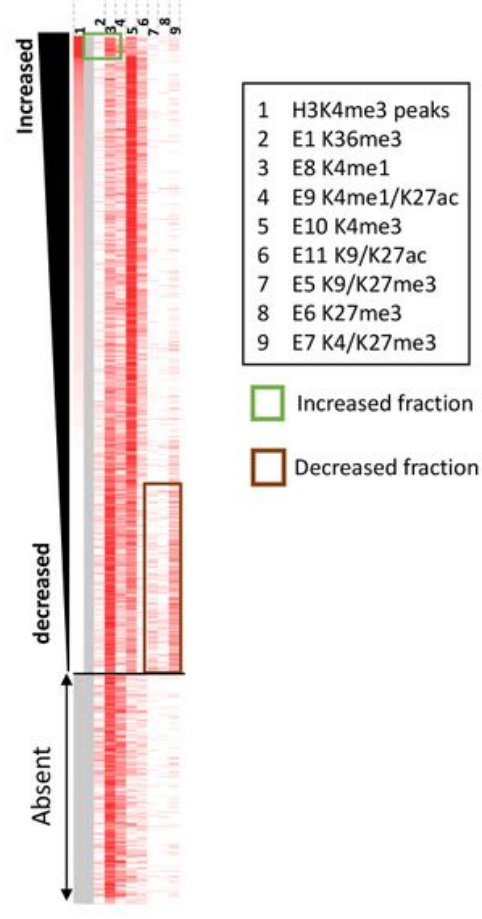

IbruR1 +3M

\section{Figure 4}

Changes in chromatin structure depending of the chromatin state. (A-G) Bar coding presentation: chromatin states as defined previously (20) (E1 and E9, H3K4me1/H3K27ac; E8, H3K4me1; E10, H3K4me3; E11, H3K27ac/H3K9ac; E5 and E6, H3K27me3; E7, H3K27me3/H3K4me1/3) have been attributed a colour code depending of their overlap with identified H3K4me3 peaks in IbruR1 and/or IbruR3 samples (red = present, white = absent). H3K4me3 peaks have been ranked based on fold-change between (A) TN 7d, (B) IbruR3 2M, (C) IbruR3 6M, (D) IbruR3 9M, (E) IbruR3 33M, (F) IbruR1 and (G) IbruR1 +3M compared to lbruR3 BT. 
A
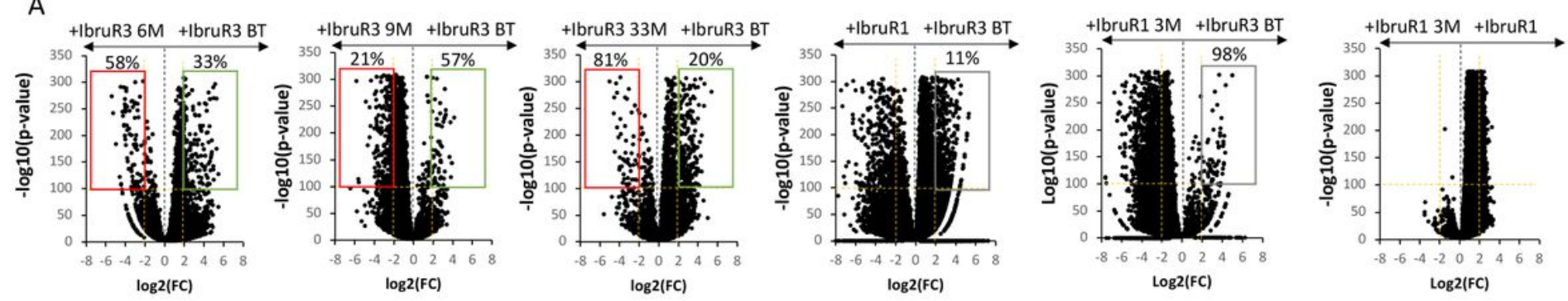

$\log 2(\mathrm{FC})$

$\log 2(\mathrm{FC})$

B

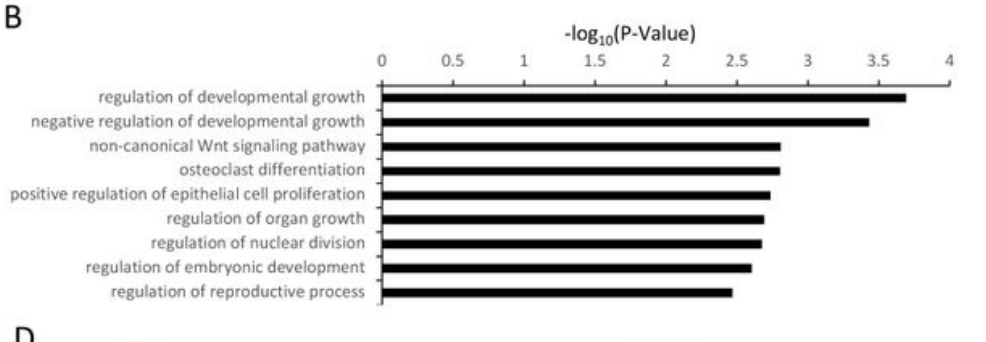

C

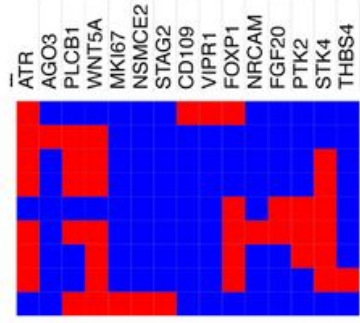

Association $\square$ or no association with the specified biological process osteoclast differentiation non-canonical Wnt signaling pathway regulation of embryonic development regulation of reproductive process regulation of organ growth regulation of developmental growth negative regulation of developmental growth positive regulation of epithelial cell proliferation regulation of nuclear division
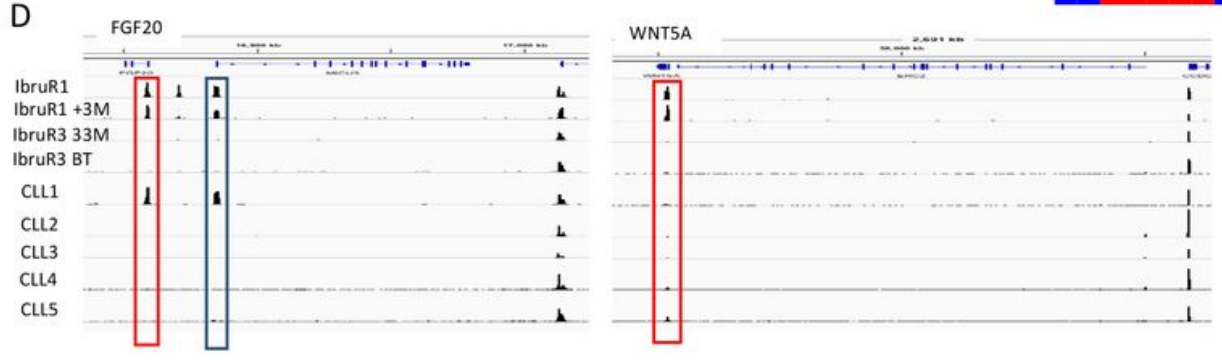

\section{Figure 5}

H3K4me3 peak intensity variation showed a significant degree of time dependent conservation. (A) Differential H3K4me3, as presented in Fig. 1 and defined as log2(H3K4me3 fold-change) and log10(-pvalue) between IbruR3 before treatment and at 6, 9, 33 months and IbruR1 as well as between IbruR1 and IbruR1 +3 months. Each H3K4me3 peak was associated with its closest gene. The percentage of peaks conserved at all time points $6 \mathrm{M}, 9 \mathrm{M}$ and $33 \mathrm{M}$ are indicated for the decreased (red rectangle) and increased (green rectangle) fractions. Similarly, the percentage of peaks conserved in both IbruR1 and IbruR1 $+3 \mathrm{M}$ are indicated for the increased (grey rectangle) fractions. (B-C) Biological processes enriched at genes associated with differentially regulated H3K4me3 peaks (IbruR1 compared to 5 CLL samples from patients which received up to 7 days ibrutinib treatment (17); hypergeometric test BH-FDR < 0.05). (D) Example of differential H3K4me3 peak intensity between the analysed samples (WNT5A and FGF20). 


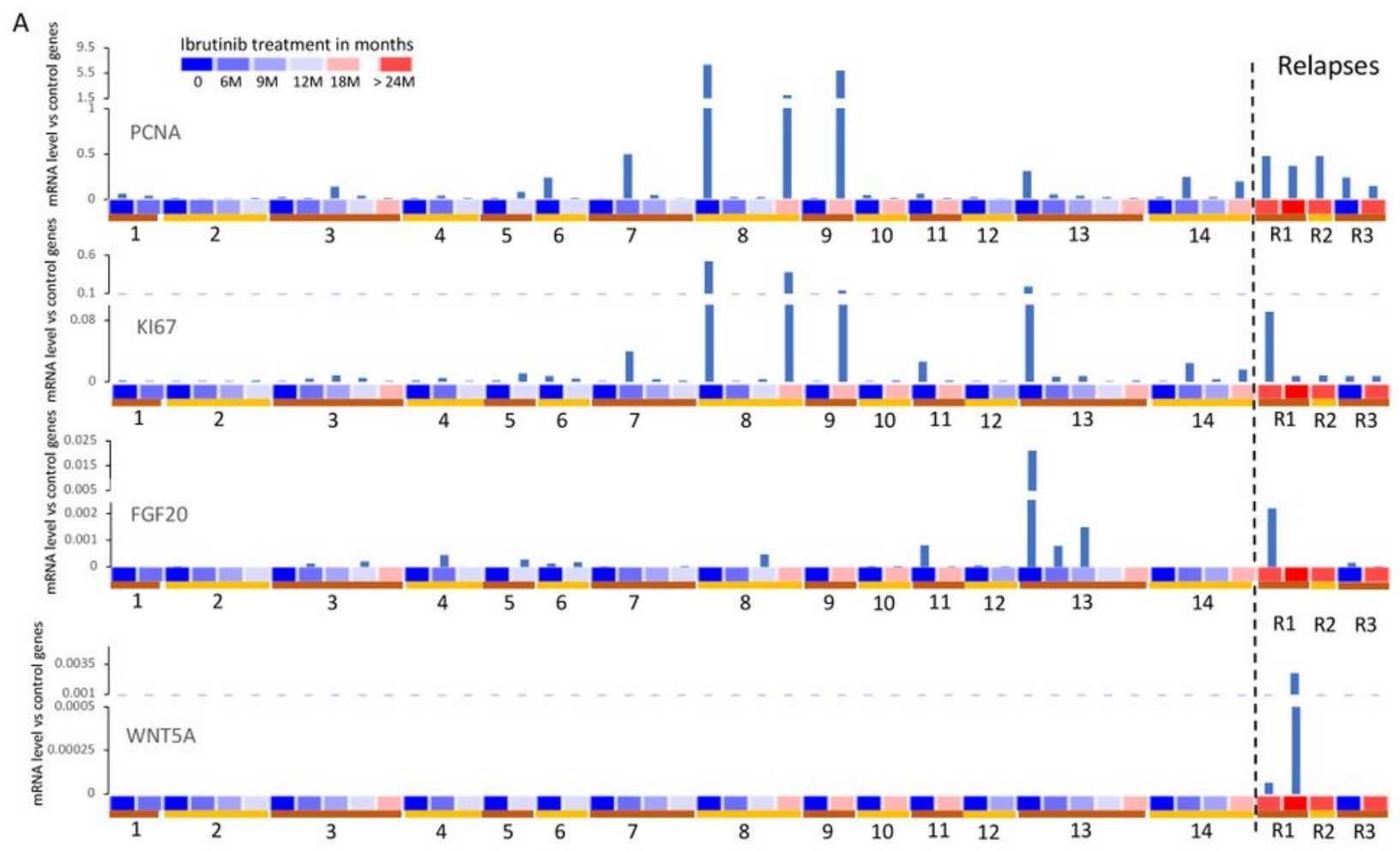

B

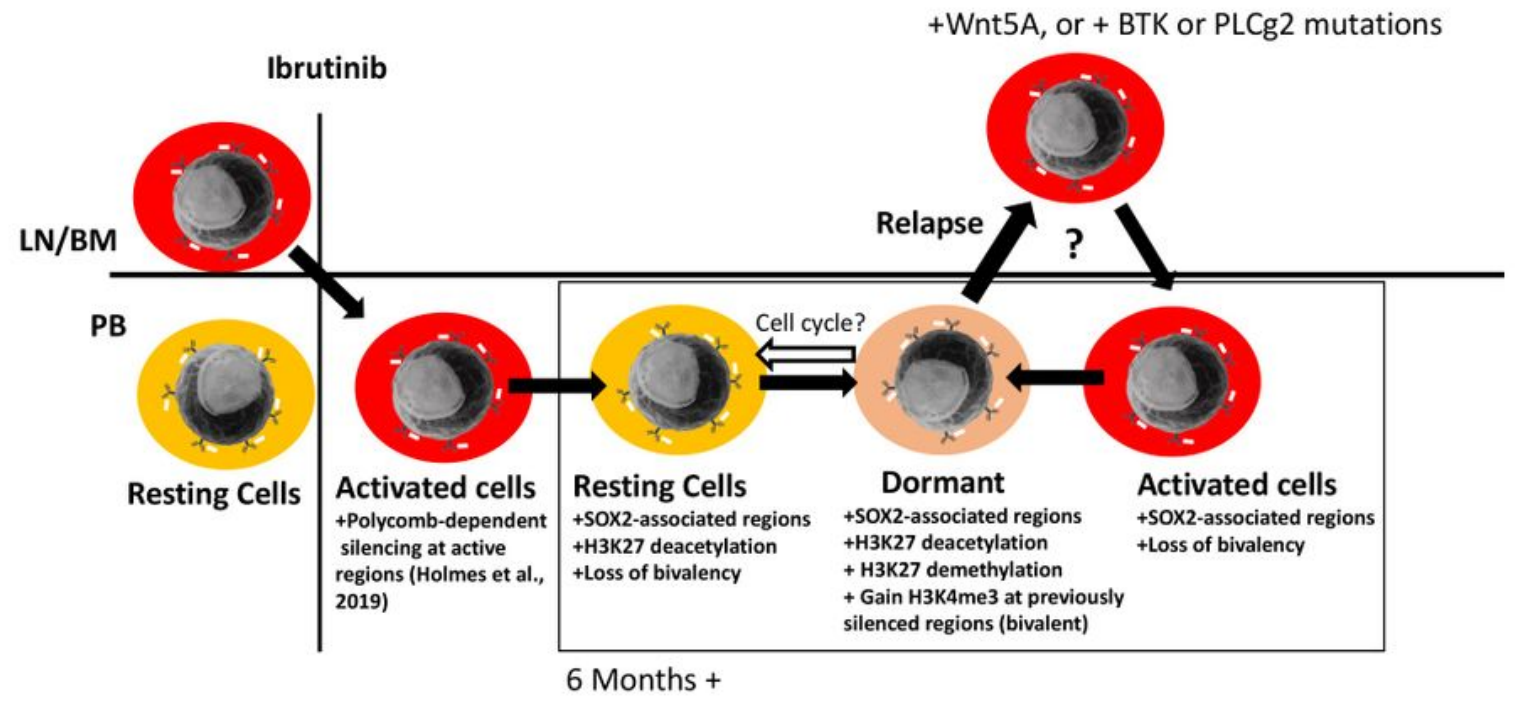

\section{Figure 6}

WNT5A is specifically expressed in IbruR1. (A) gene expression analysis of CLL cells from PB for 14 patients collected before treatment or at 6, 9,12 and 18M (as indicated on the Figure) compared to lbruR1 (R1), IbruR2 (R2) and IbruR (R3). PCNA, Ki67, FGF20 and WNT5A. Data are expressed relative to an average of 5 different control genes. (B) Simplified model highlighting different level of transcriptional activity in CLL cells on ibrutinib. 


\section{Supplementary Files}

This is a list of supplementary files associated with this preprint. Click to download.

- Supplementaryfigures.pdf 